\title{
INFLUENCIA DE LOS FACTORES TOPOGRÁFICOS EN LOS PROCESOS DE PIPING, MURCIA (ESPAÑA)
}

\author{
A. ROMERO DÍAZ*，F. ALONSO SARRÍA, A. SÁNCHEZ SORIANO
}

Departamento de Geografía, Universidad de Murcia, Campus de la Merced, 30001 Murcia.

RESUMEN. Las causas de formación de los procesos de piping, que se han descrito habitualmente, son diversas: mecánicas, químicas o biológicas, y en particular destacan las relacionadas con la presencia de sodio en el suelo y la existencia de gradientes hidráulicos. Este trabajo tiene como objetivo principal el análisis de las características topográficas, tanto de las parcelas como de los pipes y cuantificar el volumen de sedimentos que ha sido exportado por los mismos.

Se han estudiado 8 zonas con procesos de piping, localizadas en la cuenca del río Mula. El área fue aterrazada para el cultivo y abandonada desde hace 30-40 años. Se han analizado 137 parcelas de las cuales el $71 \%$ presenta procesos de piping. Los resultados muestran que la mayor o menor presencia de piping $y$ de pérdidas de suelo se concentra en determinadas zonas. Se ha observado una elevada correlación entre volumen de suelo perdido y extensión de los procesos de piping; entre superficie afectada por piping y longitudes, profundidades y anchuras medias de los pipes; entre tamaño de la parcela y grado de desarrollo del piping; entre la altura de las terrazas de cultivo y la profundidad máxima de los pipes; entre la longitud de las vaguadas, los gradientes topográficos y la intensidad de piping.

En definitiva, si los factores topográficos favorecen de algún modo la presencia de pipes, se podrían establecer recomendaciones para el diseño de parcelas, de manera que, en lo posible, minimizaran el riesgo de ocurrencia de procesos de piping en las áreas inicialmente favorables.

\section{Influence of topographic factors on piping processes, Murcia (Spain)}

ABSTRACT. Usually, different mechanical, chemical or biological causes of piping development have been described, particularly those related to the hydraulic gradients and the presence of sodium into the soil. The main purpose of this paper is to analyze the topographic characteristicsof both the pipes and the agricultural plots where they appear, and to estimate the volume of sediment yield. 
Eight different zones, located in the Mula River basin, were studied. The slopes were terraced for cultivation and abandoned 30-40 years ago. 137 plots were selected for sampling, $71 \%$ of which showed piping processes. The results obtained show a concentration of pipes in certain areas. There is a high correlation between the volume of soil loss and the extent of piping; between the area affected by piping and the average length, depth and width of pipes; between the size of the plot and the degree of piping development; between the height of the terrace walls and the maximum depth of pipes; and, finally, between gradients and piping intensity.

If topographic factors favour the presence of pipes, some plot levelling recommendations could be defined in order to minimize the risk of piping processes in the most susceptible areas.

Palabras clave: piping, erosión, campos aterrazados en abandono, factores topográficos, medios semiáridos.

Key words: piping, erosion, abandoned terraced fields, topographical factors, semiarid environment.

*Correspondencia: Departamento de Geografía, Universidad de Murcia, Campus de la Merced, 30001 Murcia. E. mail: arodi@um.es

\section{Introducción}

Los procesos de erosión subsuperficial (piping o erosión en túnel) se han identificado en numerosas regiones del mundo, pero en particular tienen una gran presencia en medios semiáridos (Bryan y Jones, 1997), como es el caso de la Región de Murcia.

La erosión en túnel o piping se atribuye generalmente a flujos subsuperficiales concentrados que pueden originarse al introducirse el agua a través de grietas de desecación o pequeñas fracturas de las rocas. El desarrollo predominante de piping se ha constatado en suelos desarrollados sobre litologías margosas con altos niveles de sodio que pueden dispersar rápidamente las arcillas, haciendo los suelos más vulnerables a la erosión y creando conductos subsuperficiales, en ocasiones de grandes dimensiones (Rengasamy et al, 1984; Naidu et al., 1995; Faulkner et al., 2003). Cuando los conductos subsuperficiales se amplían, los techos de las cavidades se desploman produciéndose colapsos que generan, en ocasiones, amplias cárcavas de paredes verticales.

Otro factor importante en el desarrollo de los procesos de piping es la presencia de gradientes hidráulicos (Parker, 1964, Faulkner et al., 2000). Los gradientes hidráulicos pueden ser naturales, pero también creados por el hombre, como es el caso de la construcción de terrazas de cultivo. El abandono de los campos de cultivo aterrazados en los 

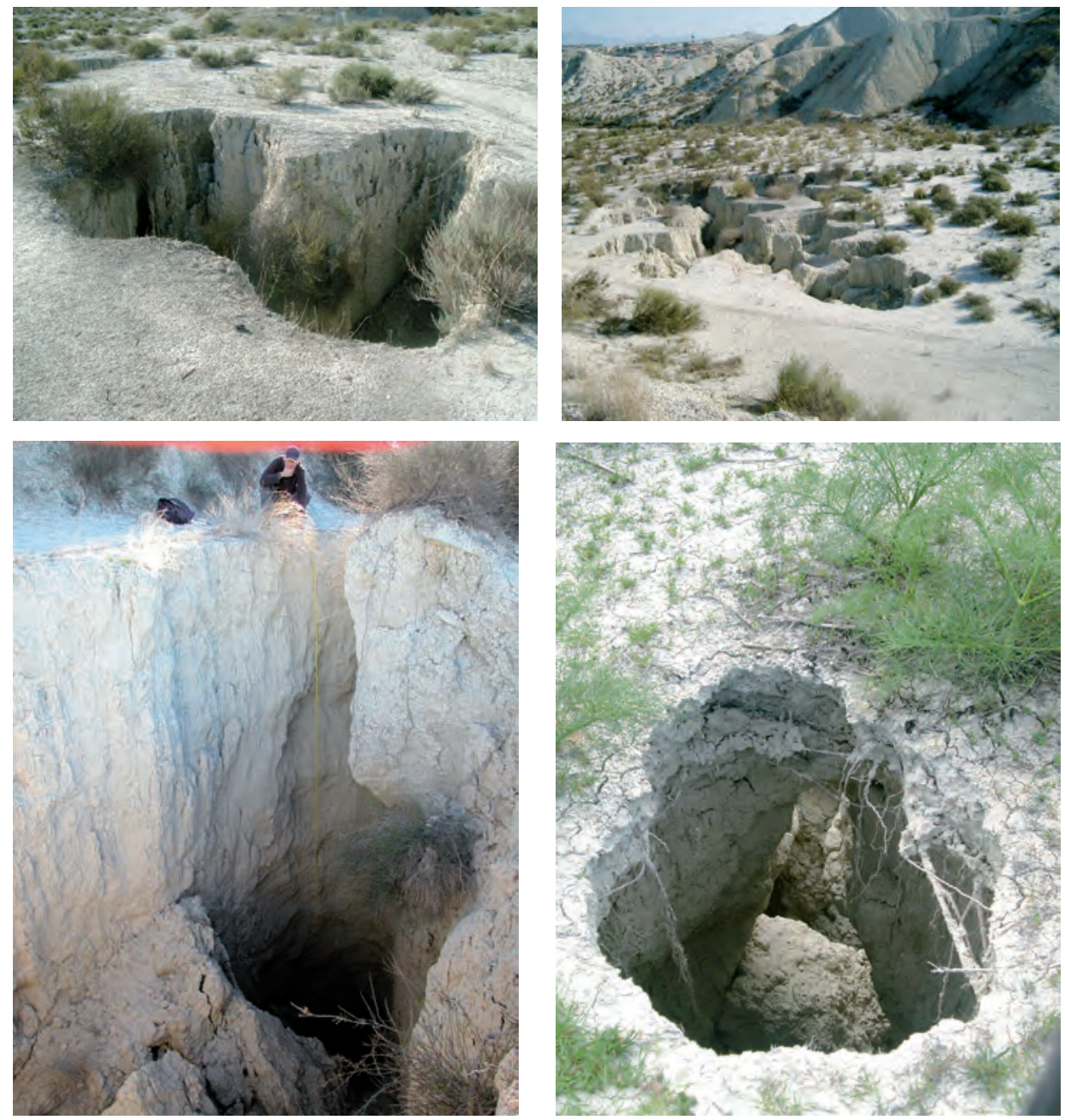

Figura 1. Algunos ejemplos de pipes en las diferentes zonas de estudio.

últimos años, en general en toda el área mediterránea y en particular en la Región de Murcia, ha propiciado el desarrollo de importantes procesos de piping (Romero Díaz et al., 2007) (Fig. 1).

Se han formulado diversas hipótesis sobre los procesos que inician los pipes y una de ellas apunta a la existencia de una zona saturada desarrollada en el frente del terraplén, por lo que el pipe comienza desde abajo; y su expansión se justifica por el agua que fluye a través de pequeños canales preexistentes en el suelo (Watt, 1991). Otras causas esgrimidas por otros autores y que se han constatado en el área estudiada de la Cuenca de Mula, además del gradiente hidráulico, son: diferencias de textura y estructura a diferentes 
profundidades, presencia de sales solubles y minerales de la arcilla que favorecen los procesos de disolución y formación de grietas de retracción (López Bermúdez y Romero Díaz, 1989; Sánchez Soriano et al., 2003).

Los procesos de piping se han observado tanto en paisajes naturales como antrópicos, en diferentes climas, litologías y depósitos, así como bajo diferentes usos del suelo y cubierta vegetal. No obstante, en medios semiáridos alcanzan un gran desarrollo y es donde se encuentran las formas mayores (Bryan y Jones, 1997). En la Región de Murcia son numerosas las áreas en las se han identificado estos procesos, Romero Díaz et al. (2009) cifran en 67 las principales áreas de afección, aunque son muchas más si se consideran las superficies más pequeñas.

Uno de los lugares más importantes por el desarrollo del piping se sitúa en la Cuenca de Mula, y en él se han seleccionado varias áreas que han sido objeto de un minucioso estudio tanto de las propiedades físico-químicas de sus suelos a distintas profundidades, como de las características morfológicas de las áreas en donde se encuentran. En un primer estudio (Romero Díaz et al., 2007) se constató la relación existente entre las variaciones físico-químicas de los suelos a distintas profundidades y la génesis de los pipes, así como la relación entre la altura de las terrazas y la profundidad de los mismos.

Este trabajo tiene como principal objetivo el análisis de las características topográficas de las áreas en donde se localizan los pipes, su posible relación con la aparición o desarrollo y las pérdidas de suelo que estos procesos producen.

\section{2. Área de estudio}

El área de estudio se localiza en la cuenca del río Mula en el término municipal de Campos del Río (Murcia) y ocupa una extensión aproximada de $2 \mathrm{~km}^{2}$. Se trata de un área de badland con un importante desarrollo de cárcavas y barrancos (Fig. 2), que descienden con un notable gradiente $(15 \%)$ hacia el cauce del río Mula. Gran parte de las vaguadas fueron aterrazadas y usadas como tierras de cultivo de cereal primero, y de almendros después en algunas de ellas, hasta aproximadamente los años setenta o principios de los ochenta del siglo pasado. A partir de estos años se produjo un progresivo abandono y el deterioro irreversible de estos campos de cultivo, en los cuales el proceso de piping se encuentra en estado muy avanzado.

Los suelos se corresponden con Regosoles calcáricos. La vegetación natural existente, tiene un escaso carácter protector y esta constituida principalmente por Salsola genistoides, Lygeum spartum, Asparagus sp., Moricandia arvensis, Thimelaea hirsuta y Artemisia sp. Las características climáticas son semiáridas, con una temperatura media de $17^{\circ} \mathrm{C}$, unas precipitaciones medias anuales en torno a los $300 \mathrm{~mm}$ (pero con una elevada irregularidad y con frecuentes episodios de lluvia de carácter torrencial) y una Evapotranspiración potencial de 900 mm (Romero Díaz y López Bermúdez, 1985). 


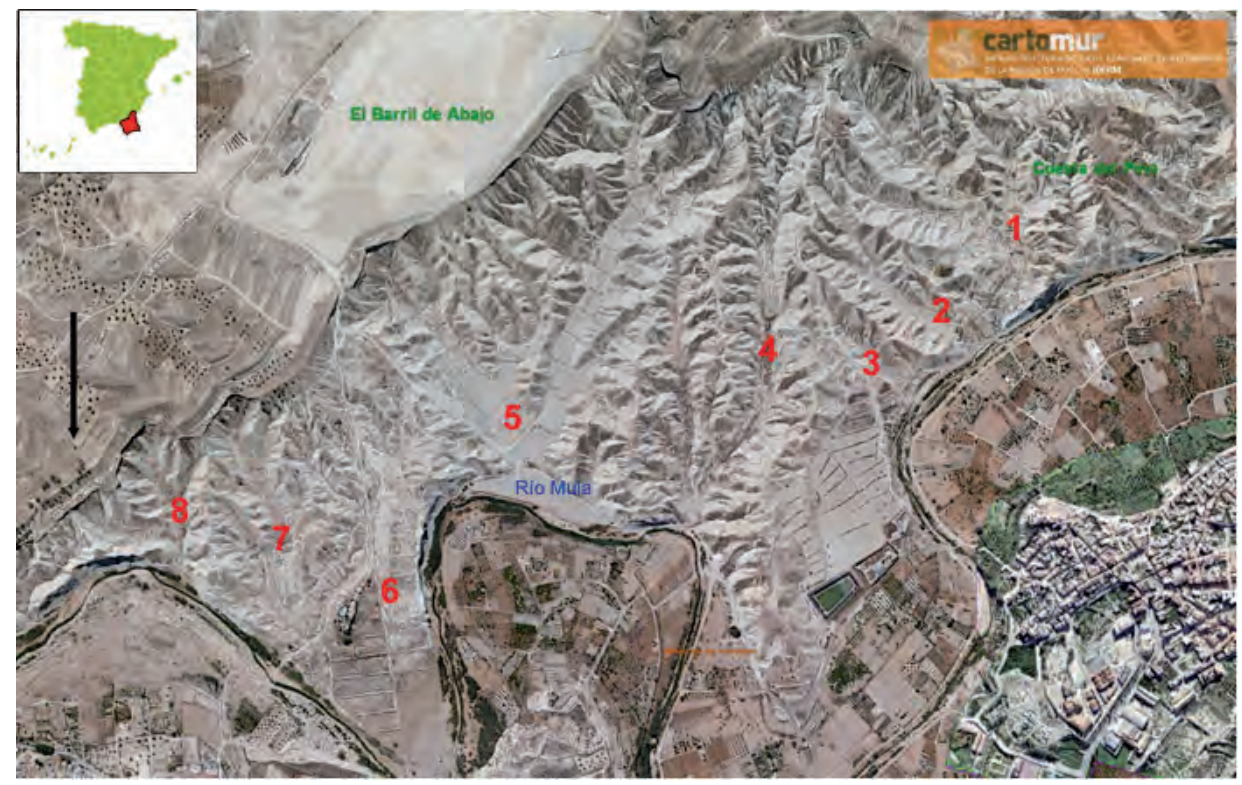

Figura 2. Localización de las áreas de estudio. Campos del Río, Murcia. Imagen obtenida de Cartomur.com.

En el área estudiada, se han individualizado 8 zonas (Fig. 2), en las que se han estudiado numerosos parámetros topográficos y morfológicos, y estimado las tasas de erosión producidas por estos procesos. Con la excepción de la zona 6 , todas ellas se corresponden con vaguadas aterrazadas y anteriormente en cultivo. La zona 6 , se trata de una ladera roturada y aterrazada para su puesta en cultivo, en espera de las aguas del trasvase Tajo-Segura, pero que nunca llegaron y, por ello, estas tierras nunca se llegaron a cultivar.

\section{Métodos}

El estudio del piping, se ha realizado a partir de minuciosos reconocimientos de campo, cartográficos e interpretación de fotografías aéreas. Se han medido: pendientes de las laderas; superficies de las parcelas; profundidades, longitudes y superficies de los pipes; altitudes entre las diferentes terrazas de cultivo; superficie afectada por piping en cada parcela, etc., con el fin de relacionar estos datos con los diferentes procesos que caracterizan la aparición y el desarrollo del fenómeno. Mediante la cubicación de los pipes y el cálculo de la densidad de los suelos se ha estimado el volumen de suelo perdido como consecuencia de estos procesos (Fig. 3). Hay que mencionar que las medidas de volúmenes y tasas de erosión obtenidas, sin duda, son inferiores a la realidad, debido a que la medición completa de los conductos que comunican los pipes en profundidad ha sido imposible de realizar. 


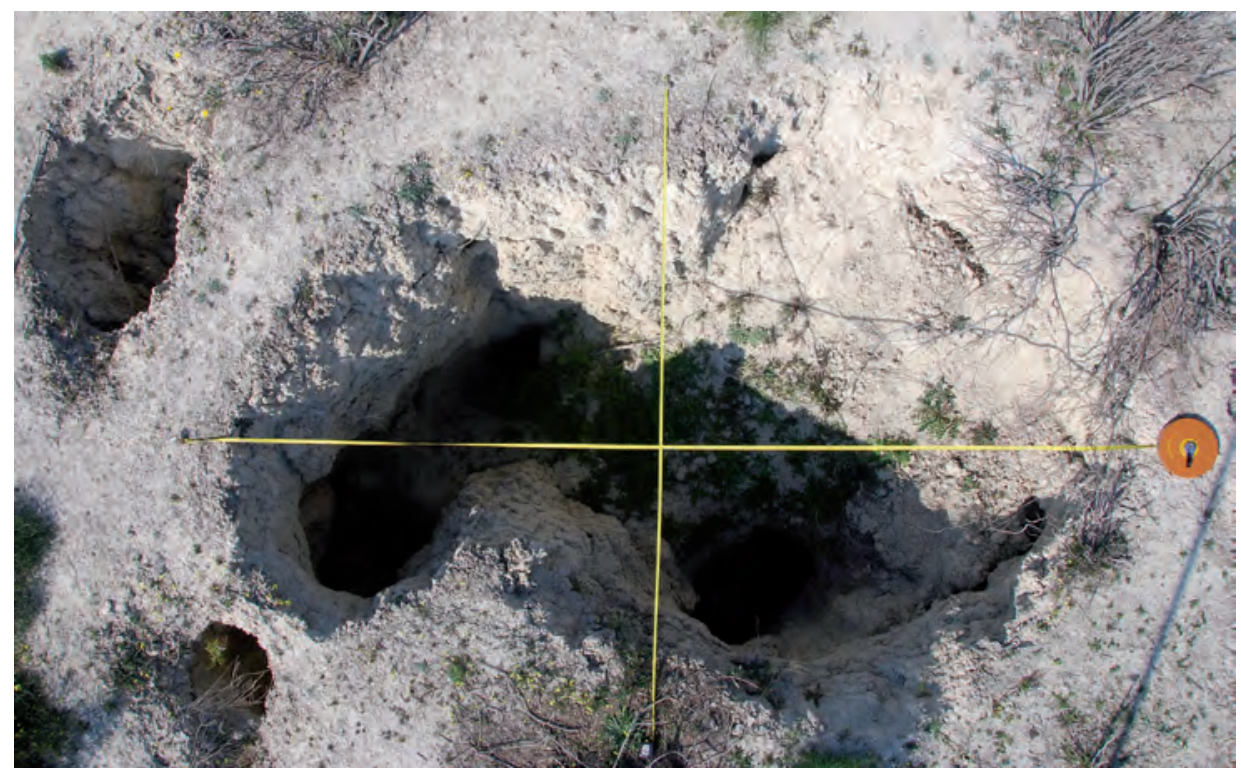

Figura 3. Foto vertical de un pipe.

Para los estudios cartográficos han sido de gran ayuda los mapas de líneas y ortofotomapas a escala 1:5.000 de la Hoja de Mula (912) del servicio de cartografía de la Comunidad Autónoma de la Región de Murcia, así como la web www.cartomur.com, que pone a disposición del usuario no sólo la cartografía topográfica, sino también las ortoimágenes de distintos años.

En el área de estudio se identificaron ocho zonas donde los procesos de piping estaban presentes, numerándolas de este a oeste (Fig. 2), e identificando el número total de parcelas existente (con o sin piping). Todas las zonas se caracterizan por ser campos de cultivo abandonados ubicados en las vaguadas de los badlands y que descienden hacia el río Mula. Las zonas 1, 2, 4 y 8 tienen su nivel de base en el curso del río.

\section{Resultados y discusión}

\section{1. Características de las parcelas}

En las diferentes áreas de estudio se han analizado 137 parcelas, de las cuales 97 mostraron procesos de piping, que se corresponden con el $71 \%$ del total de las parcelas (Tabla 1). Respecto a la superficie de las parcelas afectadas, ésta representa el $63 \%$ de la superficie total. Con excepción de las zonas 7 y 8, en las que todas sus parcelas están afectadas por piping, el resto de zonas tiene un menor número. La superficie media del 


\begin{tabular}{|c|c|c|c|c|c|c|c|c|c|c|}
\hline 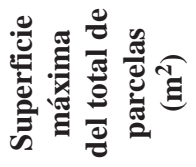 & $\begin{array}{l}\stackrel{8}{\sim} \\
\text { }\end{array}$ & 옹 & $\stackrel{n}{\varrho}$ & $\begin{array}{l}\stackrel{+}{9} \\
\text { d) }\end{array}$ & $\begin{array}{l}\stackrel{0}{\circ} \\
\stackrel{+}{+}\end{array}$ & $\begin{array}{l}\infty \\
\infty \\
m\end{array}$ & $\stackrel{8}{8}$ & $\stackrel{n}{\stackrel{2}{*}}$ & $\begin{array}{l}n \\
\tilde{n} \\
n\end{array}$ & \\
\hline 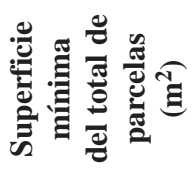 & $\stackrel{n}{\stackrel{n}{n}}$ & હે & $\stackrel{\circ}{7}$ & 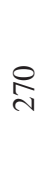 & 운 & $\stackrel{2}{\infty}$ & 8 & $\frac{n}{n}$ & $\stackrel{\theta}{m}$ & \\
\hline 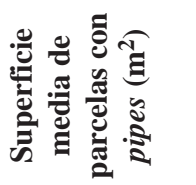 & 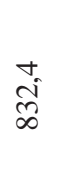 & $\begin{array}{l}0 \\
\stackrel{0}{0} \\
\stackrel{0}{0}\end{array}$ & $\frac{a}{a}$ & 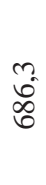 & $\begin{array}{l}0 \\
\hat{n} \\
\infty \\
\infty\end{array}$ & $\begin{array}{l}0 \\
i \\
\infty \\
\stackrel{0}{0}\end{array}$ & $\begin{array}{l}0 \\
\text { \&े } \\
\text { mे }\end{array}$ & $\begin{array}{l}0 \\
\stackrel{\infty}{+}\end{array}$ & $\begin{array}{c}\vec{m} \\
\stackrel{2}{n}\end{array}$ & \\
\hline 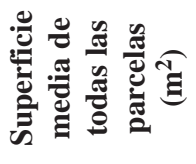 & 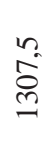 & $\frac{6}{2}$ & 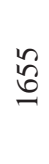 & $\stackrel{n}{\stackrel{n}{N}}$ & $\begin{array}{l}\stackrel{n}{n} \\
\text { na }\end{array}$ & $\begin{array}{l}\stackrel{2}{~} \\
\text { הે }\end{array}$ & ஓ & $\stackrel{f}{f}$ & $\hat{m}_{\infty}$ & \\
\hline 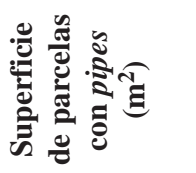 & $\begin{array}{l}\infty \\
\stackrel{\infty}{ \pm} \\
I\end{array}$ & $\stackrel{\text { p }}{\sim}$ & $\stackrel{n}{\stackrel{2}{2}}$ & 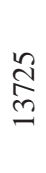 & $\begin{array}{l}\text { ్ } \\
\stackrel{\text { }}{+}\end{array}$ & $\begin{array}{l}\text { నু } \\
\text { న }\end{array}$ & 웅 & $\begin{array}{l}\tilde{ర} \\
\widetilde{b}\end{array}$ & $\frac{\text { लำ }}{2}$ & 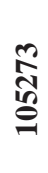 \\
\hline 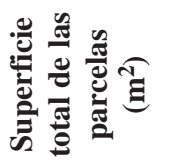 & $\begin{array}{l}\text { ते } \\
\stackrel{\text { సे }}{ }\end{array}$ & 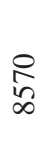 & $\begin{array}{l}\stackrel{2}{\infty} \\
0 \\
0\end{array}$ & $\begin{array}{l}\text { ๙ } \\
\text { ஜn }\end{array}$ & $\begin{array}{l}\curvearrowright \\
\text { సે }\end{array}$ & 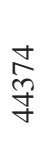 & 웟 & $\begin{array}{l}\tilde{D} \\
\text { గ్ర }\end{array}$ & ڤ̊. & $\frac{\hat{\kappa}}{2}$ \\
\hline 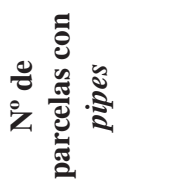 & $\vec{\sim}$ & $N$ & $\infty$ & సి & 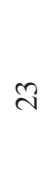 & $\nabla$ & 0 & 9 & 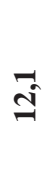 & $\hat{a}$ \\
\hline 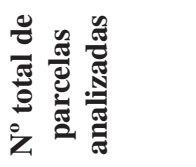 & i & $m$ & 든 & $\vec{\lambda}$ & ৯े & ્ & 0 & $\stackrel{m}{=}$ & $\sqrt{5}$ & $\hat{2}$ \\
\hline$\stackrel{\tilde{\Xi}}{\stackrel{\tilde{N}}{\mathbf{N}}}$ & - & $N$ & $m$ & $\forall$ & $n$ & 0 & $r$ & $\infty$ & 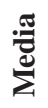 & $\stackrel{\text { సేّ }}{0}$ \\
\hline
\end{tabular}


Tabla 2. Altura de las parcelas

\begin{tabular}{|c|c|c|c|c|}
\hline Zona & $\begin{array}{c}\text { Altura media } \\
\text { entre todas las } \\
\text { parcelas (m) }\end{array}$ & $\begin{array}{c}\text { Altura media } \\
\text { entre parcelas } \\
\text { con pipes (m) }\end{array}$ & $\begin{array}{c}\text { Altura media } \\
\text { entre parcelas } \\
\text { con pipes }(\sigma)\end{array}$ & $\begin{array}{c}\text { Altura máxima } \\
\text { entre parcelas (m) }\end{array}$ \\
\hline 1 & 1,8 & 2,1 & 0,6 & 3,5 \\
\hline 2 & 1,8 & 2,5 & 0,5 & 4,2 \\
\hline 3 & 2,5 & 2,9 & 1,4 & 5,4 \\
\hline 4 & 1,5 & 1,7 & 0,8 & 4 \\
\hline 5 & 2,2 & 2,3 & 0,9 & 5 \\
\hline 6 & 1,8 & 1,9 & 0,1 & 4,5 \\
\hline 7 & 1,9 & 1,9 & 1,1 & 2,8 \\
\hline 8 & 1,7 & 1,7 & 0,6 & $\mathbf{3 , 1 5}$ \\
\hline Media & $\mathbf{1 , 9}$ & $\mathbf{2 , 1}$ & $\mathbf{1 , 0}$ & \\
\hline
\end{tabular}

total de las parcelas por zonas es de $873,7 \mathrm{~m}^{2}$, oscilando desde $190 \mathrm{~m}^{2}$ de media en las parcelas de la zona 7 a los $1765 \mathrm{~m}^{2}$ de la zona 1 . Las parcelas más pequeñas tienen una superficie de $100 \mathrm{~m}^{2}$ y las más grandes superan los $5000 \mathrm{~m}^{2}$. Si se consideran solamente las parcelas que contienes pipes la superficie media por zonas aumenta, siendo de $1353 \mathrm{~m}^{2}$; no obstante, hay que mencionar que existe una variabilidad grande con una desviación típica de 696. Para las superficies con pipes, la zona 7 sigue siendo la que tiene menor superficie media, pero la superficie se reduce notablemente en la zona 4. Se ha comprobado como las áreas en las que se dan las mayores pérdidas de suelo (t/ha) se corresponden con las zonas de menor superficie y mayor pendiente.

Altura de las parcelas. La altura media entre todas las parcelas estudiadas es de $1,9 \mathrm{~m}$, oscilando entre $1,7 \mathrm{~m}$ y $2,5 \mathrm{~m}$ (Tabla 2). Por el contrario, en las parcelas que han desarrollado procesos de piping la altura media se eleva a 2,1 m y en la mayoría de las zonas los valores de altura entre las distintas terrazas también es superior. Hay que indicar que en 5 de las 8 zonas estudiadas se dan parcelas con una altura superior a $4 \mathrm{~m}$ y en dos de ellas se superan los 5. La altura de las terrazas, en ocasiones, puede ser un importante factor de formación de pipes, debido al gradiente hidráulico existente. Los valores medios de alturas de terrazas por zonas no se corresponden con las zonas en donde el desarrollo de pipes es mayor, para comprobar si existe una relación entre desarrollo de piping y altura entre terrazas es necesario analizar el conjunto total de parcelas.

Longitud de las parcelas. La longitud media de todas las parcelas, como en el caso de la superficie, también es ligeramente superior a la longitud de las parcelas con 


\begin{tabular}{|c|c|c|c|c|c|c|c|c|c|c|c|c|c|c|c|c|c|c|c|c|}
\hline 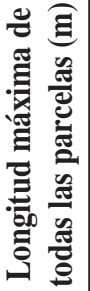 & $\stackrel{n}{n}$ & Ð & $\stackrel{n}{7}$ & $\infty$ & 8 & $\stackrel{\infty}{=}$ & D & 2 & N & & 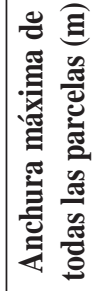 & 6 & $\stackrel{\infty}{\sim}$ & $\stackrel{n}{0}$ & $\curvearrowright$ & 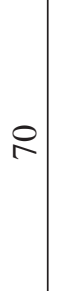 & $\bar{n}$ & $\frac{n}{2}$ & $\begin{array}{l}n \\
2 \\
2\end{array}$ & $\begin{array}{l}n \\
\text { ñ } \\
7\end{array}$ \\
\hline 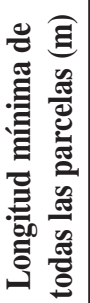 & $n$ & $\begin{array}{l}n \\
n \\
n\end{array} \mid$ & $a$ & $\begin{array}{l}n \\
\pm \\
\pm\end{array}$ & $\stackrel{\curvearrowright}{.}$ & $\begin{array}{l}n \\
\tilde{n} \\
n\end{array} \mid$ & $\stackrel{0}{=}$ & $\simeq$ & $\begin{array}{l}n^{2} \\
2\end{array}$ & & 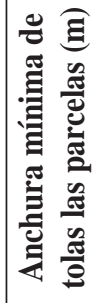 & $a$ & 每 & $\infty$ & $=$ & $\cong$ & $\stackrel{\sim}{\circ}$ & ๙́a & $\simeq$ & 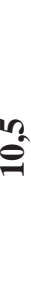 \\
\hline 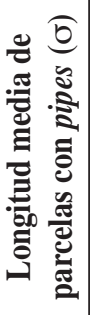 & $\hat{0}$ & $\begin{array}{l}\dot{\sigma}_{-} \\
\dot{\nabla}\end{array}$ & $\begin{array}{l}0 \\
i\end{array}$ & $\hat{n}$ & $\vec{n}$ & $\begin{array}{l}\nabla^{\prime} \\
\infty\end{array}$ & $\stackrel{n}{r}$ & $\Xi$ & $\begin{array}{l}\infty \\
\pm \\
\pm\end{array}$ & 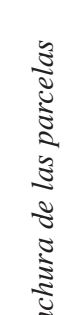 & 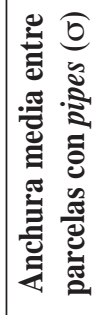 & \pm & $\stackrel{\infty}{=}$ & $\vec{m}$ & $\begin{array}{l}0 \\
\stackrel{ \pm}{ \pm}\end{array}$ & $\overrightarrow{\mathrm{N}}$ & ç. & 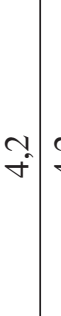 & $\begin{array}{l}\mathcal{N} \\
f\end{array}$ & $\hat{\Omega}^{1}$ \\
\hline 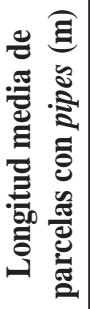 & $\stackrel{n}{\hat{N}}$ & $\mid \begin{array}{l}\infty \\
\dot{\mathbb{f}}\end{array}$ & $\begin{array}{l}\infty \\
\infty \\
\infty\end{array}$ & $\tilde{\imath}$ & $\stackrel{\infty}{\infty}$ & ○ & $\vec{\sim}$ & $\stackrel{n}{\sim}$ & $\begin{array}{l}\infty \\
\tilde{m} \\
\tilde{m}\end{array}$ & $\frac{\dot{\sigma}}{\frac{\pi}{\delta}}$ & 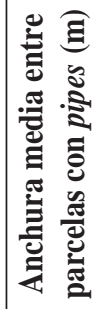 & ?. & 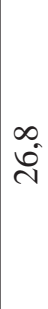 & $\begin{array}{l}m \\
g \\
q\end{array}$ & 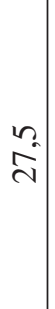 & $\underset{y}{F}$ & $m$ & 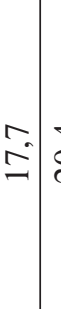 & $\begin{array}{l}\dot{J}_{0} \\
\tilde{\sim}\end{array}$ & ڤ్ \\
\hline 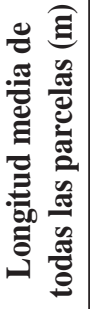 & $\begin{array}{l}\dot{ }^{\prime} \\
\stackrel{0}{\sim}\end{array}$ & $\begin{array}{l}m \\
\mathfrak{f}\end{array}$ & $\vec{\sim}$ & $\begin{array}{l}\mathfrak{N} \\
\tilde{v}\end{array}$ & $\begin{array}{l}m \\
f\end{array}$ & $\begin{array}{l}n \\
2 \\
6\end{array}$ & $\begin{array}{l}0 \\
\stackrel{\sim}{\sim} \\
\dot{\sim}\end{array}$ & $\stackrel{\infty}{\sim}$ & m. & & 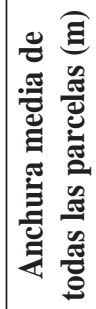 & $\stackrel{-}{\infty}$ & $\stackrel{\sim}{d}$ & $\begin{array}{l}n \\
i \\
n\end{array}$ & $\stackrel{n}{\sim}$ & $\stackrel{?}{7}$ & $\vec{m}$ & $\begin{array}{l}n \\
\infty \\
- \\
\end{array}$ & $\begin{array}{l}n \\
\tilde{2}\end{array}$ & हे \\
\hline$\stackrel{\overparen{\Xi}}{\tilde{\Xi}}$ & - & $N$ & $m$ & $\nabla$ & $n$ & 0 & $r$ & $\infty$ & 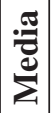 & & 織 & - & $\sim$ & $m$ & $\nabla$ & $n$ & 6 & 10 & $\infty$ & 莡 \\
\hline
\end{tabular}



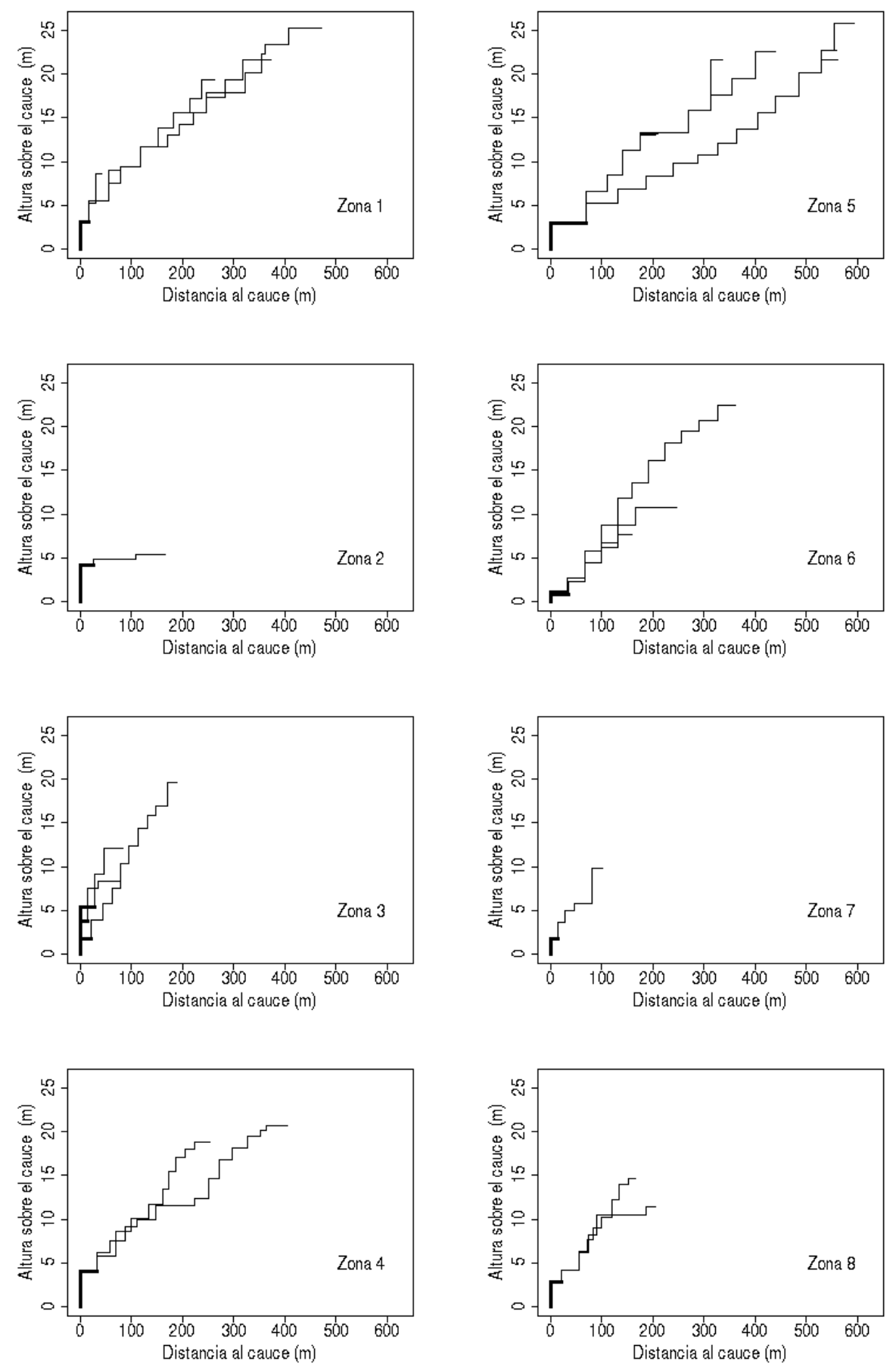

Figura 4. Perfiles topográficos de las parcelas de las distintas áreas. 
pipes, aunque cada zona se comporta de una manera diferente (Tabla 3). La media de las parcelas que tienen una longitud más pequeña se ha estimado en 13,5 $\mathrm{m}$ y la de mayor longitud en $77 \mathrm{~m}$; no obstante, es posible encontrar longitudes de parcelas de 118 y 160 $\mathrm{m}$. Es de mencionar que las zonas en donde se alcanzan las mayores longitudes, como son las zonas 5 y 6 , se corresponden con aquellas zonas que menos perdidas de suelo (t/ ha) han registrado.

Anchura de las parcelas. La anchura media de las parcelas es prácticamente igual, tanto si se considera el conjunto total o tan sólo las parcelas con pipes, siendo el valor medio de 30,7 y 30,8 respectivamente (Tabla 4). Las anchuras máximas oscilan entre 21,5 y $105 \mathrm{~m}$, y las mínimas entre 9 y $26 \mathrm{~m}$. Se observa como las zonas 7 y 8 , que son las que tienen la menor anchura media y máxima, son también unas de las zonas que registran las mayores pérdidas de suelo. No ocurre así en la zona 4 donde no parece haber relación aparente entre la anchura de las parcelas y la pérdida de suelo.

Perfiles topográficos de los sistemas de parcelas. Para observar la posible relación existente entre el desarrollo de pipes y la topografía se han representado los perfiles topográficos de los sistemas de parcelas en cada una de las zonas (Fig. 4). Como todos los perfiles se han construido con las mismas escalas en los ejes X e Y, pueden ser objeto de comparación. Prescindiendo del área 2, por el escaso número de parcelas, se observa como el área 5 es la que tiene, en conjunto, las mayores longitudes llegando a $600 \mathrm{~m}$ desde el cauce, las mayores anchuras de sus parcelas y las menores alturas entre ellas. Es de mencionar que esta zona es una de las que menos pipes tiene y que menores

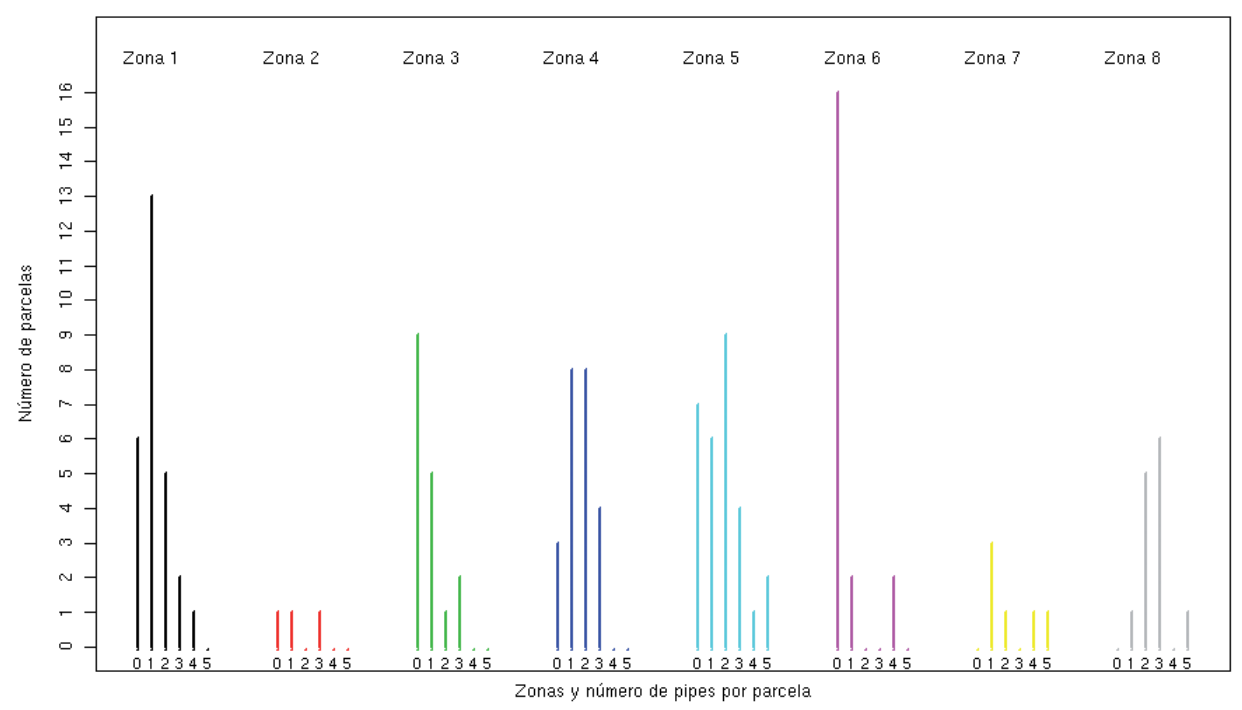

Figura 5. Número de pipes por parcela en las diferentes zonas. 
Tabla 5. Distribución de parcelas por zonas y grado de afección de piping

\begin{tabular}{|c|c|c|c|c|}
\hline Zona & $\begin{array}{c}\text { Parcelas con } \\
\text { piping }(\%)\end{array}$ & $\begin{array}{c}\text { Superficie media } \\
\text { afectada }(\%)\end{array}$ & $\begin{array}{c}\mathbf{N}^{\mathbf{0}} \text { de pipes } \\
\text { total por zona }\end{array}$ & $\begin{array}{c}\text { No de pipes } \\
\text { máximo por } \\
\text { parcela }\end{array}$ \\
\hline 1 & 80,7 & 23,7 & 33 & 29 \\
\hline 2 & 66,6 & 47 & 13 & 4 \\
\hline 3 & 47 & 24,6 & 36 & 29 \\
\hline 4 & 86,9 & 56,6 & 50 & 44 \\
\hline 5 & 79,3 & 15,2 & 10 & 10 \\
\hline 6 & 10 & 6,1 & 14 & 12 \\
\hline 7 & 100 & 46,7 & 34 & 28 \\
\hline 8 & 100 & 54,1 & $\mathbf{3 3 , 5}$ & $\mathbf{2 8 , 5}$ \\
\hline Media & $\mathbf{7 1 , 3}$ & $\mathbf{3 4 , 3}$ & $\mathbf{1 9 4}$ & \\
\hline Total & $\mathbf{1 0 0}$ & & & 43 \\
\hline
\end{tabular}

pérdidas de suelo ha registrado. Por el contrario, las zonas 7, 8, 3 y una de las vaguadas de les área 4, presentan, en conjunto, distancias al cauce menores y alturas relativas al mismo elevadas. Es de mencionar como la primera parcela de la zona 4 presenta un desnivel muy acusado cuyo nivel de base es cauce del río. En definitiva, parece existir una relación entre vaguadas cortas en longitud con importantes gradientes topográficos y desarrollo de piping.

\subsection{Características de los pipes}

Independientemente de otros factores, como pueden ser los litológicos, parece que pudiera existir en el área de estudio una distribución no aleatoria, que pudiera explicarse por factores topográficos. Así el mayor porcentaje de parcelas con desarrollo de piping se encuentra en las zonas 7, 8 y 4. Todas las parcelas de las zonas 7 y 8 tienen pipes, con una superficie de afección media elevada (46,7 y 54,3\%), junto con las zonas 4 y 2 ( 56.6 y $47 \%$ ), en torno o superior al $50 \%$ de la superficie de la parcela. El mayor número 


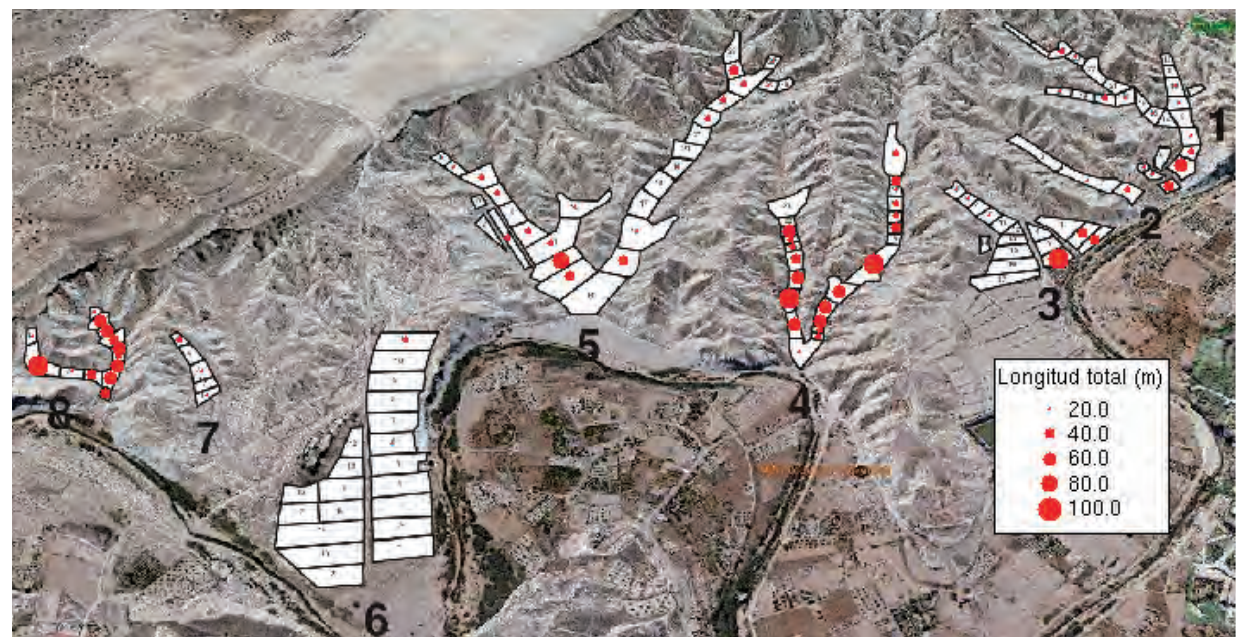

Figura 6. Longitud total de pipes por parcela.

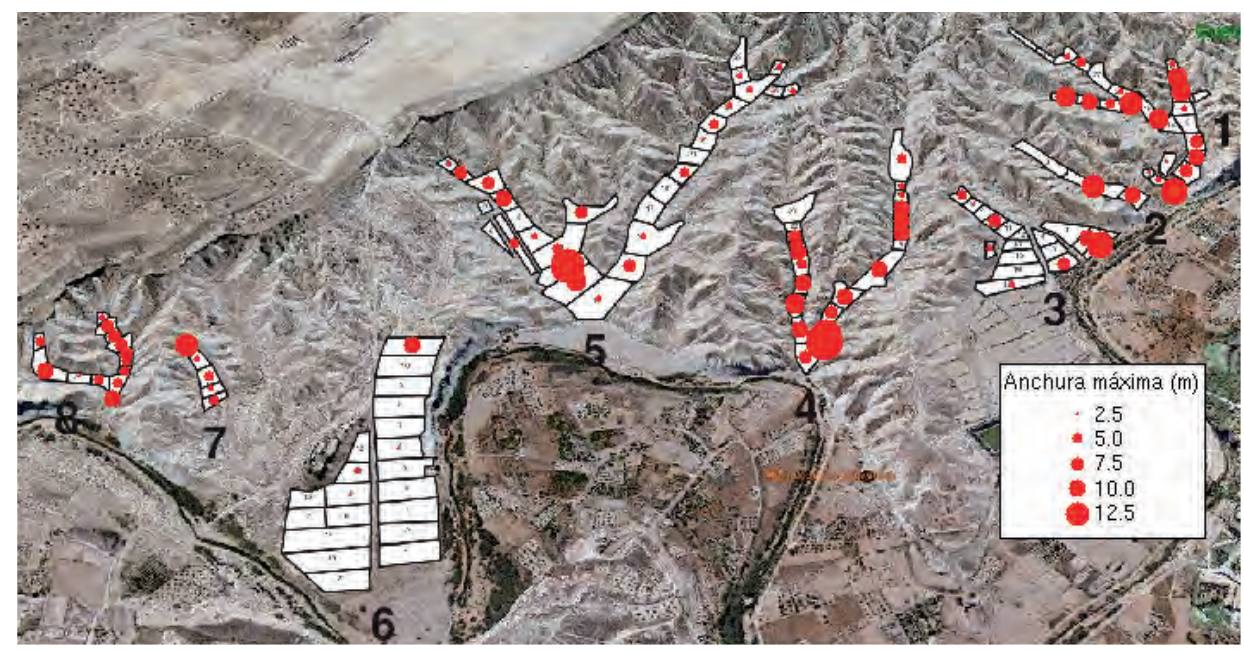

Figura 7. Anchura máxima de pipes por parcela.

de pipes no siempre se corresponde con las áreas de mayor afección superficial, pues en ocasiones, como sucede en la zona 5 se desarrolla un número elevado de pipes (50), mientras que la afección superficial tan sólo es del 15\%, debido al menor tamaño de los pipes o escasa evolución de los mismos, por el momento (Fig. 5). Por el contrario, en la zona 2, con escaso número de pipes, pero de grandes dimensiones, suponen el $47 \%$ de 


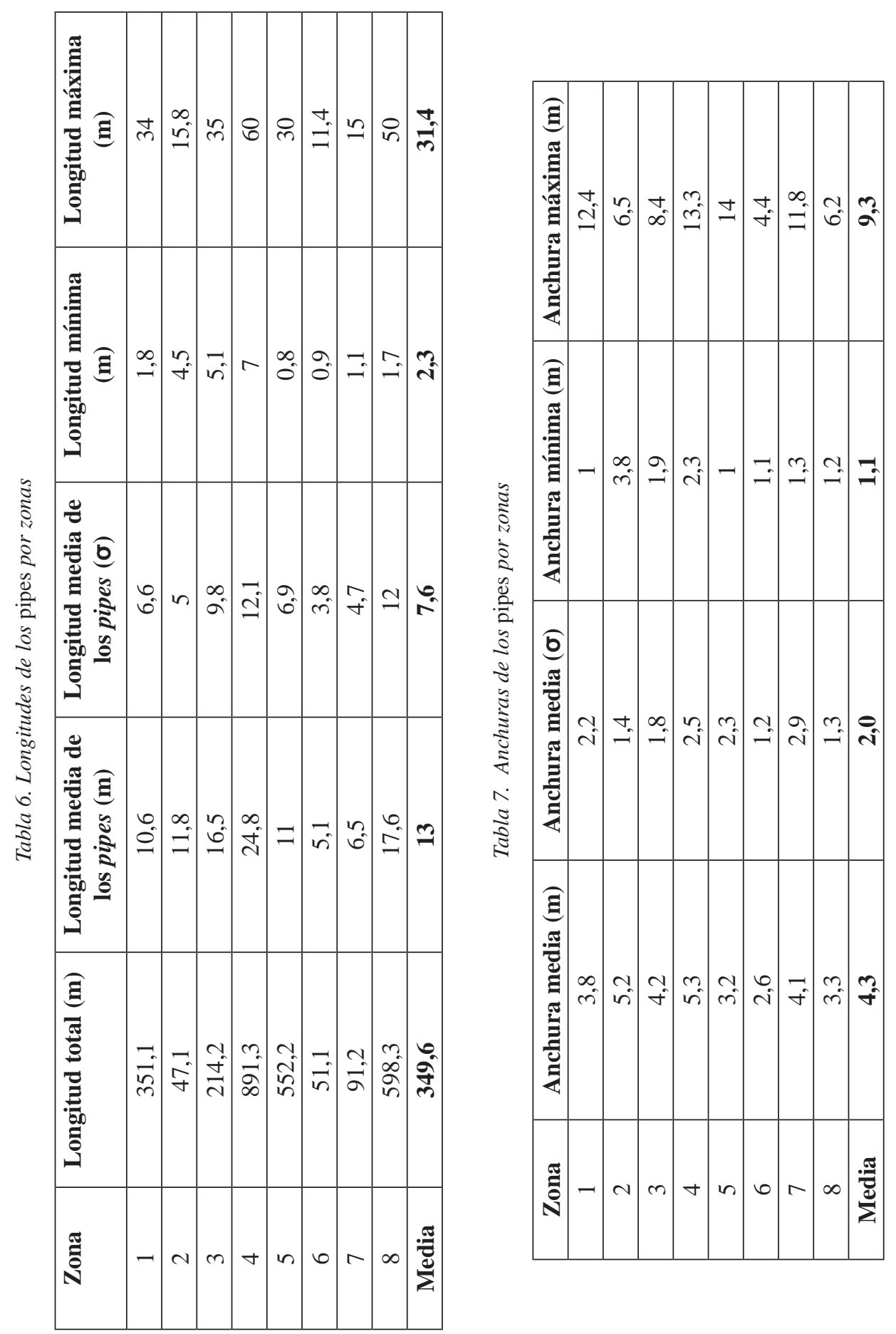


Tabla 8. Profundidad de los pipes por zonas

\begin{tabular}{|c|c|c|c|c|}
\hline Zona & $\begin{array}{c}\text { Profundidad } \\
\text { media }(\mathbf{m})\end{array}$ & $\begin{array}{c}\text { Profundidad } \\
\text { media }(\boldsymbol{\sigma})\end{array}$ & $\begin{array}{c}\text { Profundidad } \\
\text { mínima }(\mathbf{m})\end{array}$ & $\begin{array}{c}\text { Profundidad } \\
\text { máxima }(\mathbf{m})\end{array}$ \\
\hline 1 & 1,4 & 1 & 0,5 & 4,5 \\
\hline 2 & 2.1 & 0,9 & 0,8 & 2,8 \\
\hline 3 & 1.4 & 0,8 & 0,4 & 3,2 \\
\hline 4 & 2,6 & 1,1 & 0,8 & 5,6 \\
\hline 5 & 1.2 & 0,5 & 0,5 & 3 \\
\hline 6 & 0,6 & 0,3 & 0,3 & 1 \\
\hline 7 & 1,7 & 1,1 & 0,5 & 3,6 \\
\hline 8 & 1,7 & 0,8 & 0,5 & 3,2 \\
\hline Media & $\mathbf{1 , 6}$ & $\mathbf{0 , 8}$ & $\mathbf{0 , 5}$ & $\mathbf{3 , 8}$ \\
\hline
\end{tabular}

superficie afectada. La densidad mayor de pipes por parcela se encuentra también en la zona 5 y 4 , y la menor en la zona 2 (Tabla 5).

Longitud de los pipes. El desarrollo longitudinal total y medio de los pipes es mayor en la zona 4, seguida de la zona 8 (Fig. 6), que son las dos zonas donde las pérdidas de suelo evaluadas han sido mayores, aunque hay que mencionar que también en estas áreas se da la mayor variabilidad. En algunas zonas, donde los procesos de piping están muy desarrollados, los pipes parecen seguir el trazado de la red de drenaje existente con anterioridad a la construcción de las terrazas. En estas mismas zonas se han medido

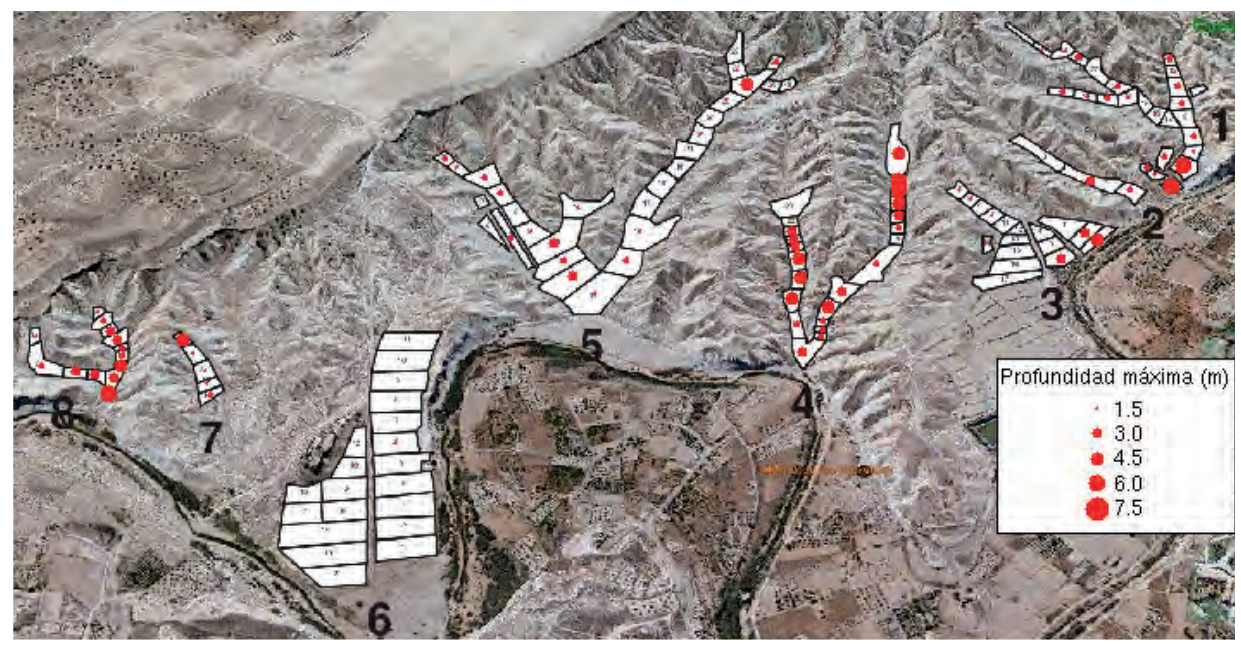

Figura 8. Profundidad máxima de pipes por parcela. 
longitudes máximas de pipes muy considerables, de 60 y 50 metros (zonas 4 y 8); la longitud mínima mayor se ha localizado también en la zona 4. Por el contrario, las longitudes mínimas se localizan en las zonas 5 y 6 , que son en las que menores pérdidas de suelo se han evaluado (Tabla 6).

Anchura de los pipes. Los pipes tienen unas anchuras medias en las diferentes zonas comprendidas entre los 2,6 y 5,3 metros (Tabla 7). Los pipes de mayor anchura media se localizan en las zonas 4, 7 y 2. Los pipes de máxima anchura se dan en las zonas 5, 4, 1 y 5, con anchuras comprendidas en estos casos entre los 12 y $14 \mathrm{~m}$; y por el contrario los de menor anchura se encuentran en las zonas 1,5 y 6. Destaca nuevamente la zona 4, con la mayor anchura media, y junto con la zona 5 con elevadas anchuras máximas de los pipes (Fig. 7).

Profundidad de los pipes. La profundidad media de los pipes en las diferentes zonas es de 1,6 metros, aunque en la zona 4 la profundidad media es de 2,6 m (Tabla 8). Es en esta zona donde también se alcanzan las profundidades máximas: 5,6 metros. Los valores de profundidades máximas medias se sitúan en 3,8 metros y los de las mínimas en 0,5. La profundidad máxima que alcanzan algunos pipes es superior a la profundidad de la parcela en la que se hayan, cuando esto ocurre es que el pipe ha alcanzado el nivel de base de la parcela inferior, o en ocasiones el nivel de base del río, si se trata de pipes localizados en la parcela mas cercana al mismo, como es el caso de las zonas $1 \mathrm{y}$ 8 (Fig. 8).

Las longitudes, anchuras y profundidades de los pipes estudiados ponen de manifiesto las grandes dimensiones que pueden alcanzan estos pipes en zonas semiáridas, diferentes de otros medios climáticos. Se ha constatado como las dimensiones de la pipes varían notablemente según el medio climático de formación. Bryan y Jones (1997) dan un promedio de diámetros de los pipes en ambientes templados de 0,15 a 0,25 m; Botschek et al. (2002) encontraron diámetros que van desde 0,05 hasta $0,30 \mathrm{~m}$ en loess de Alemania; Verachtert et al., (2010) de 0,2 m en Bégica; y Uchida et al., (2001) en los bosques japoneses miden diámetros muy pequeños (desde 0.001 hasta $0,5 \mathrm{~m}$, y a veces incluso menores de $1 \mathrm{~cm}$ ). Por el contrario, en regiones semi-áridas, los diámetros puedes ser de varios metros (Bryan y Jones, 1997; Uchida et al., 2001; Zhu, 2003), como es el caso de nuestras mediciones.

\subsection{Volumen de sedimentos evacuado por los pipes y tasas de erosión}

El volumen de suelo perdido por procesos de piping en las 8 zonas de estudio es de $25232 \mathrm{~m}^{3}$ o de $32801 \mathrm{t}$. La media por zonas alcanza valores de $239,4 \mathrm{~m}^{3}$ o $311,1 \mathrm{t}$. Si se considera la relación volumen/superficie, los valores medios son de $0,4 \mathrm{~m}^{3} / \mathrm{m}^{2}$ o de 5132 t/ha. Y finalmente las tasas de erosión calculadas dan unas pérdidas de suelo medias de 146,6 t/ha/año y de 128,3 t/ha/año, según el cálculo se haga para 35 o 40 años de periodo de abandono de estos campos y suponiendo que el inicio del piping tuvo lugar como consecuencia de este abandono. 


\begin{tabular}{|c|c|c|c|c|c|c|c|c|c|c|c|}
\hline 起导 & 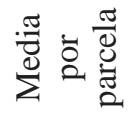 & ळ) & जे & $\stackrel{R}{2}$ & స్ & $\vec{\sim}$ & $\begin{array}{l}\infty \\
i\end{array}$ & กิ & กิ & 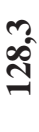 & \\
\hline 氖 & 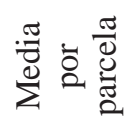 & తి & ले & $\stackrel{\infty}{\infty}$ & กิ & $\frac{n}{\sim}$ & ñ & 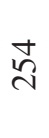 & \begin{tabular}{l} 
กี \\
\multirow{2}{n}{}
\end{tabular} & 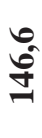 & \\
\hline$\stackrel{\text { gٌ }}{\text { S }}$ & 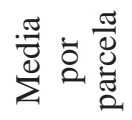 & $\begin{array}{l}- \\
\infty \\
n \\
n\end{array}$ & $\begin{array}{c}n \\
6 \\
n\end{array}$ & $\frac{\infty}{2}$ & \begin{tabular}{l}
$n$ \\
ñ \\
\multirow{2}{\pm}{}
\end{tabular} & 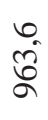 & $\stackrel{0}{=}$ & $\frac{n}{2}$ & $\begin{array}{l}n \\
\infty \\
\infty \\
\infty\end{array}$ & กิ & \\
\hline 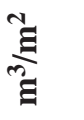 & 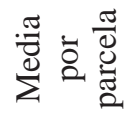 & $\tilde{o}^{n}$ & $\overrightarrow{0}$ & గn & $\cong$ & $\overrightarrow{0}$ & $\stackrel{0}{0}$ & $\hat{0}$ & $\hat{0}$ & $\stackrel{\nabla_{\sigma}}{\sigma_{0}}$ & \\
\hline \multirow{2}{*}{ } & 0 & ڤి & $\begin{array}{l}\infty \\
= \\
=\end{array}$ & $\frac{n}{8}$ & $\begin{array}{l}\infty \\
\text { ले }\end{array}$ & సิ & $\begin{array}{l}\infty \\
\stackrel{N}{N}\end{array}$ & $\stackrel{+}{i}$ & 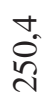 & ชี่ & \\
\hline & 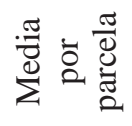 & $\begin{array}{l}\dot{v} \\
\infty \\
n\end{array}$ & $\stackrel{\infty}{8}$ & $\begin{array}{l}0 \\
\infty \\
\infty \\
\infty\end{array}$ & $\hat{\sigma}$ & 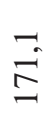 & $\begin{array}{l}\stackrel{a}{+} \\
\text { in }\end{array}$ & 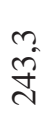 & $\frac{\infty}{\forall}$ & $=$ & \\
\hline \multirow{2}{*}{$\widetilde{\xi}$} & b & $\begin{array}{l}\text { चే } \\
\text { d }\end{array}$ & ळे & $\frac{\infty}{n}$ & $\hat{n}$ & $\hat{\sigma}^{\infty}$ & $a$ & $\begin{array}{l}\infty \\
\infty \\
\infty\end{array}$ & $\begin{array}{l}0 \\
\text { a }\end{array}$ & हె & \\
\hline & 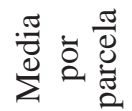 & $\frac{a}{d}$ & $\stackrel{m}{m}$ & $\stackrel{\tilde{n}}{\hat{n}}$ & $\begin{array}{l}n \\
\infty \\
n\end{array}$ & $\frac{0}{n}$ & $a$ & $\tilde{\infty}$ & $\frac{0}{i}$ & $\hat{\text { ते }}$ & \\
\hline \multirow{2}{*}{ 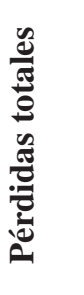 } & - & त̃ & $\frac{0}{\infty}$ & \begin{tabular}{l}
0 \\
$\infty$ \\
0 \\
\multirow{0}{+}{}
\end{tabular} & $\frac{n}{\sqrt{n}}$ & $\begin{array}{l}\dot{v} \\
\text { ñ } \\
\text { nे }\end{array}$ & å & $\begin{array}{l}\hat{a} \\
\text { In }\end{array}$ & $\begin{array}{l}m \\
\text { m } \\
\dot{H}\end{array}$ & $\frac{\text { ํ. }}{8}$ & 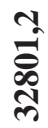 \\
\hline & $\mathrm{m}^{\mathrm{E}}$ & 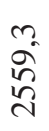 & $\begin{array}{l}0 \\
6 \\
6\end{array}$ & $\stackrel{2}{\circ}$ & 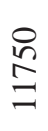 & กู & $\stackrel{0}{\circ}$ & ป̂ & 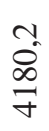 & $\begin{array}{l}\theta_{0}^{0} \\
\frac{10}{n}\end{array}$ & $\begin{array}{l}\infty \\
\text { ñ } \\
\text { ñ }\end{array}$ \\
\hline & 苂 & - & $N$ & $m$ & $\forall$ & $n$ & 0 & $r$ & $\infty$ & $\sum^{\stackrel{\pi}{g}}$ & है \\
\hline
\end{tabular}




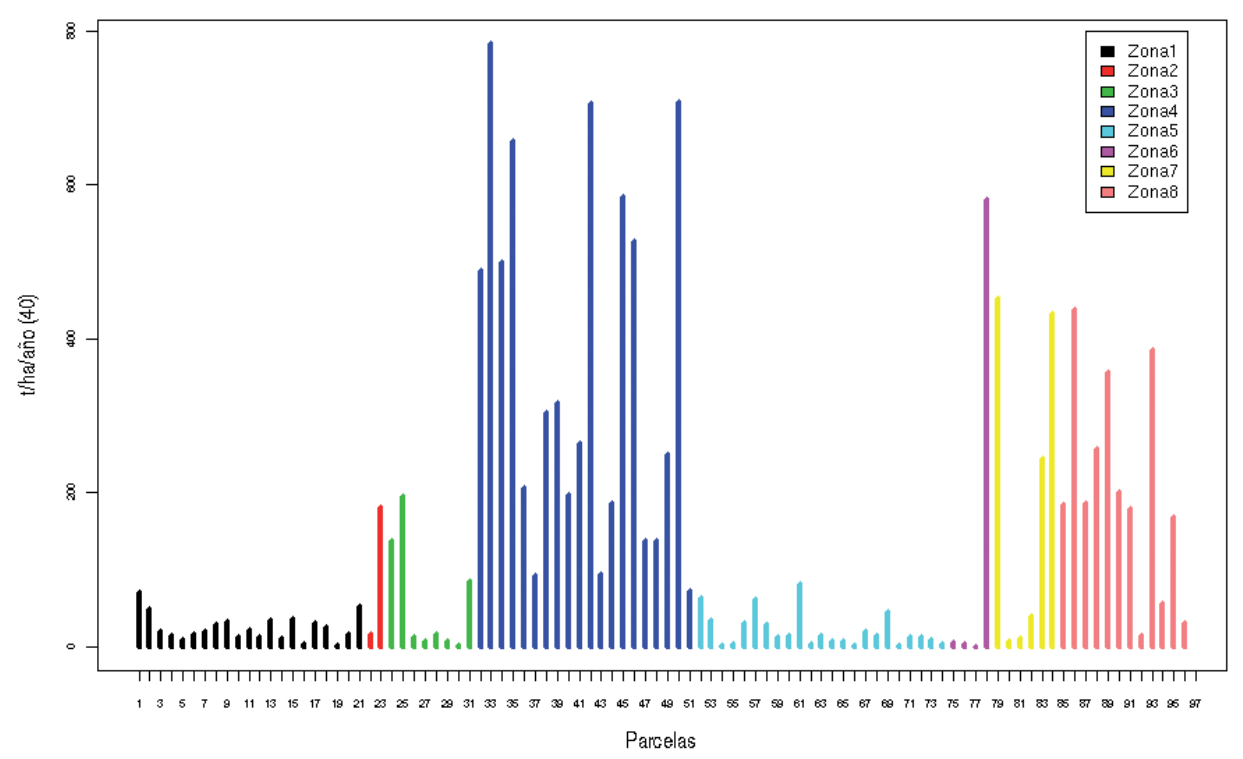

Figura 9. Tasa de erosión (t/ha/año) para 40 años en las diferentes parcelas estudiadas (se ha eliminado el valor 1415 t/ha/año).

Aunque estos valores de pérdidas de suelo ya son muy notables, los valores medios enmascaran las elevadas tasas alcanzadas en algunas áreas, como son las zonas 4, 7 y 8. Es de destacar la zona 4 con unas pérdidas de suelo de $11750 \mathrm{~m}^{3} \mathrm{o} 763,7 \mathrm{t}$ y una tasa media de erosión de 413 o de 361 t/ha/año, según se considere el periodo de abandono (Tabla 9). A pesar de que los volúmenes de suelo perdido y las tasas de erosión alcanzan notables cantidades, sin duda, son inferiores a la realidad, debido a que la medición completa de los conductos que comunican los pipes en profundidad ha sido imposible de realizar.

Si se atiende a las tasas de erosión calculadas para un periodo de abandono de 40 años, el 35\% de las parcelas presenta tasas de erosión superiores a 100 t/ha/año, el 24\% superiores a 200 t/ha/año y en el 13\% de las parcelas se superan las 500 t/ha/año (Fig. 9).

Los valores de tasas de erosión obtenidos ponen en evidencia, una vez más, que este tipo de procesos generan elevadas pérdidas de suelos (Poesen et al., 1996; Torri et al., 2002), generando efectos muy significativos fuera del área donde se producen, contribuyendo a elevar notablemente la cantidad de sedimentos que pueden llevar los cauces (Faulkner, 2006). Por otro lado, las superficies agrícolas afectadas se convierten rápidamente en improductivas e irrecuperables en la mayoría de los casos, pasando a ser "tierras baldías". Valores mucho mas pequeños de tasas de erosión en piping, entre 2,3 y 4,6 t/ha/año se han calculado para medios mucho más húmedos como es el caso del centro de Bélgica (Verachtert et al., 2010). 


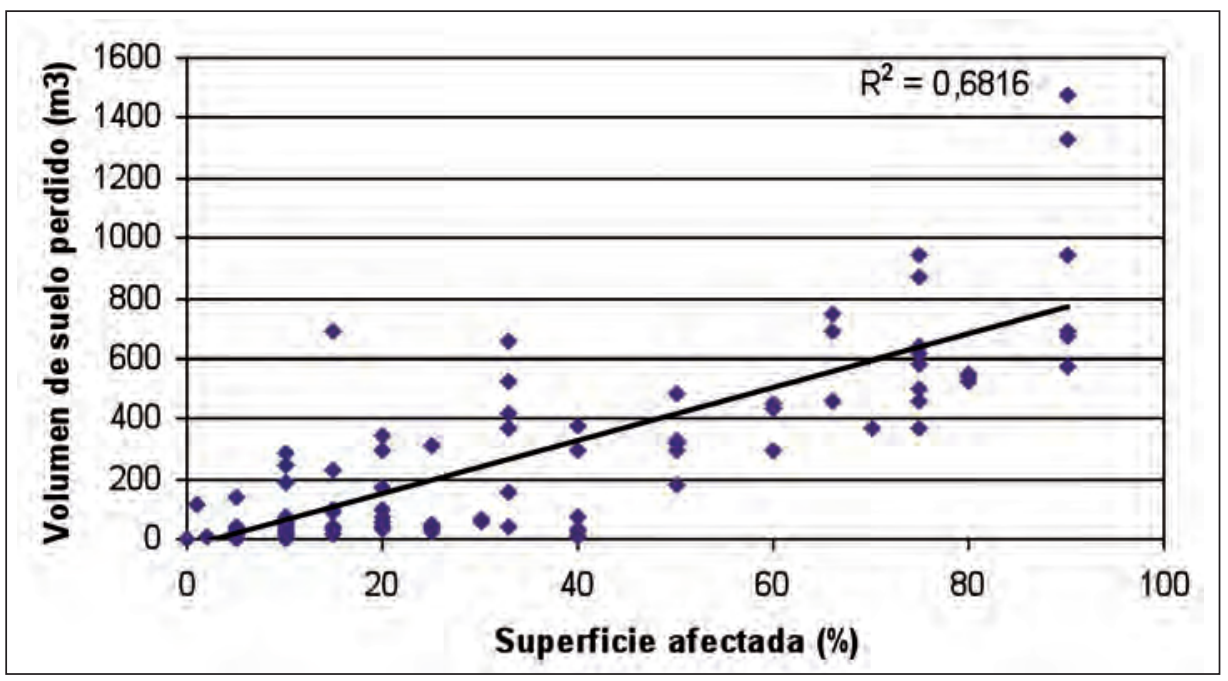

Figura 10. Relación entre superficie afectada por piping y pérdidas de suelo.

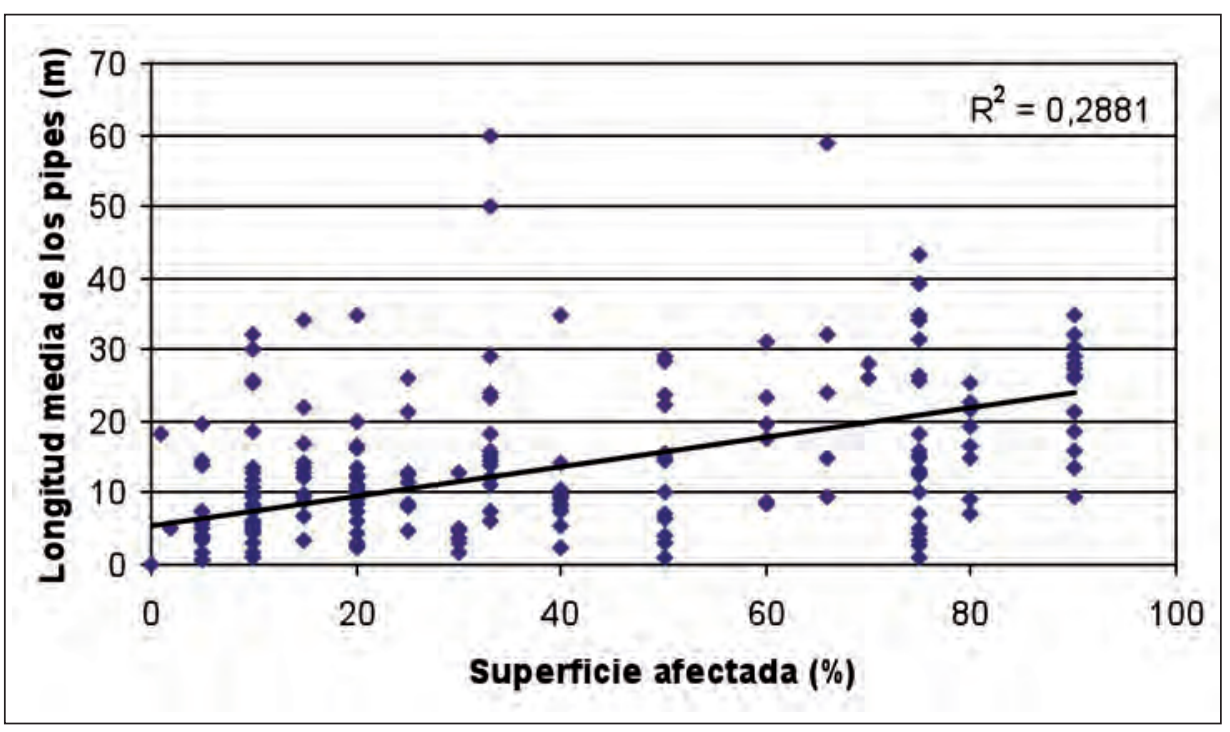

Figura 11. Relación entre superficie afectada por piping y longitud media de los pipes. 


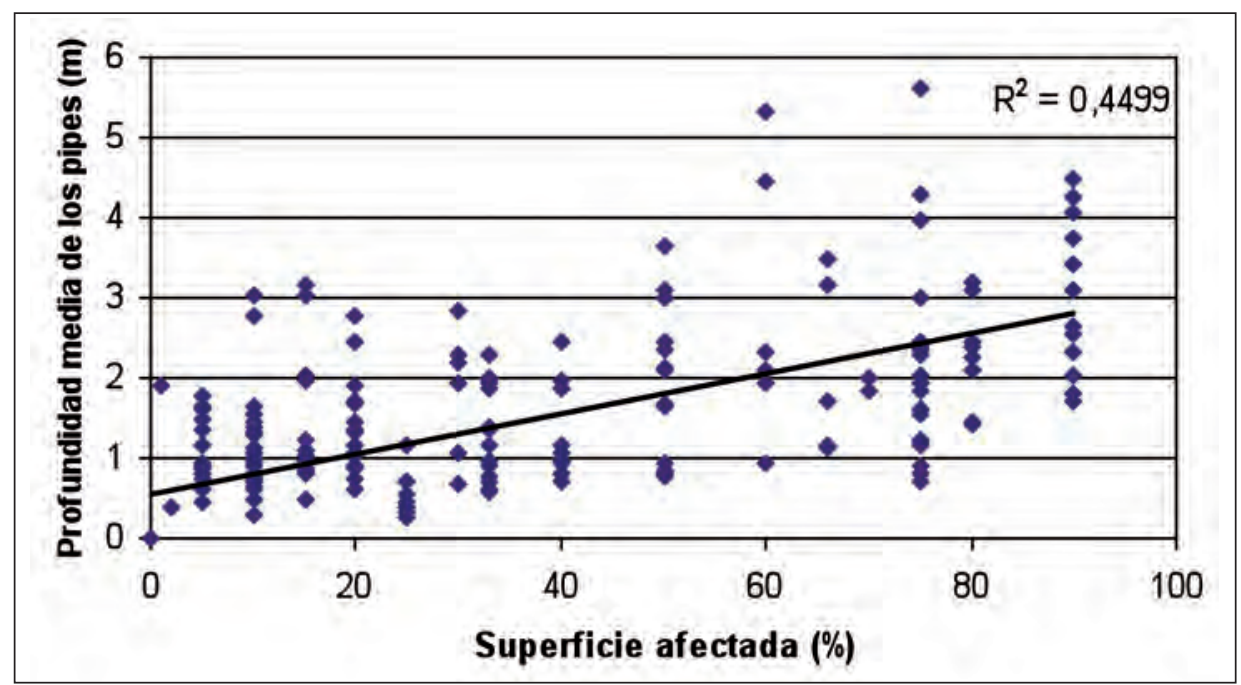

Figura 12. Relación entre superficie afectada por piping y profundidad media de los pipes.

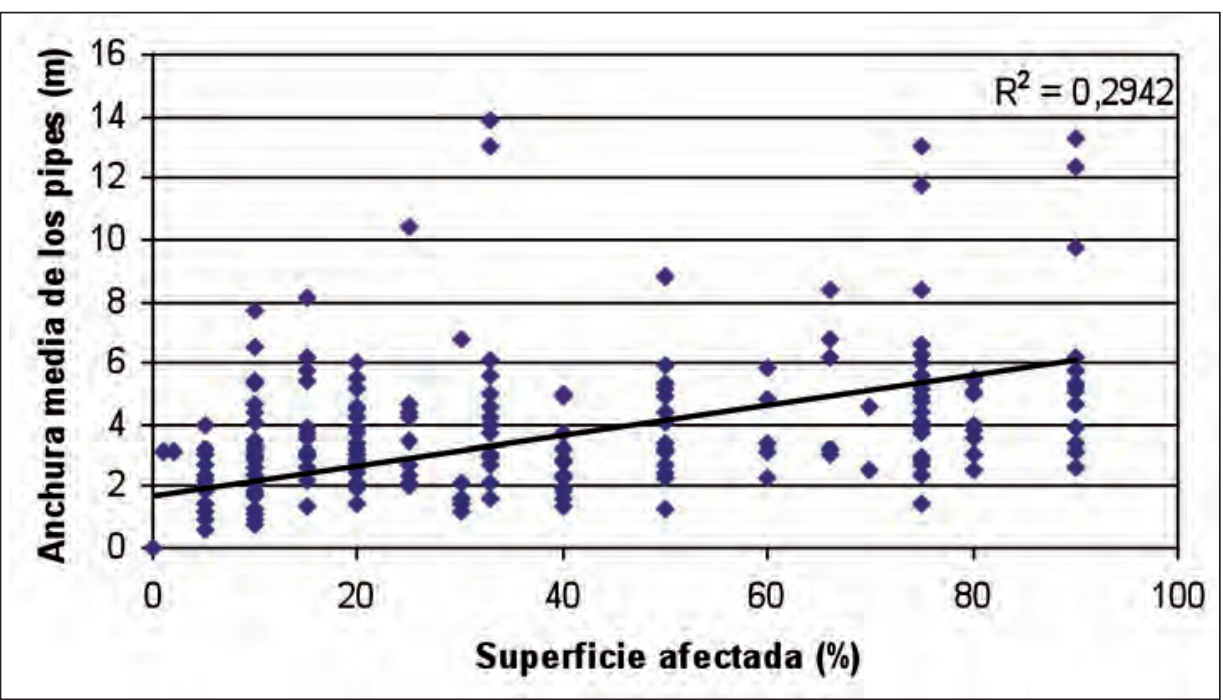

Figura 13. Relación entre superficie afectada por piping y anchura media de los pipes. 


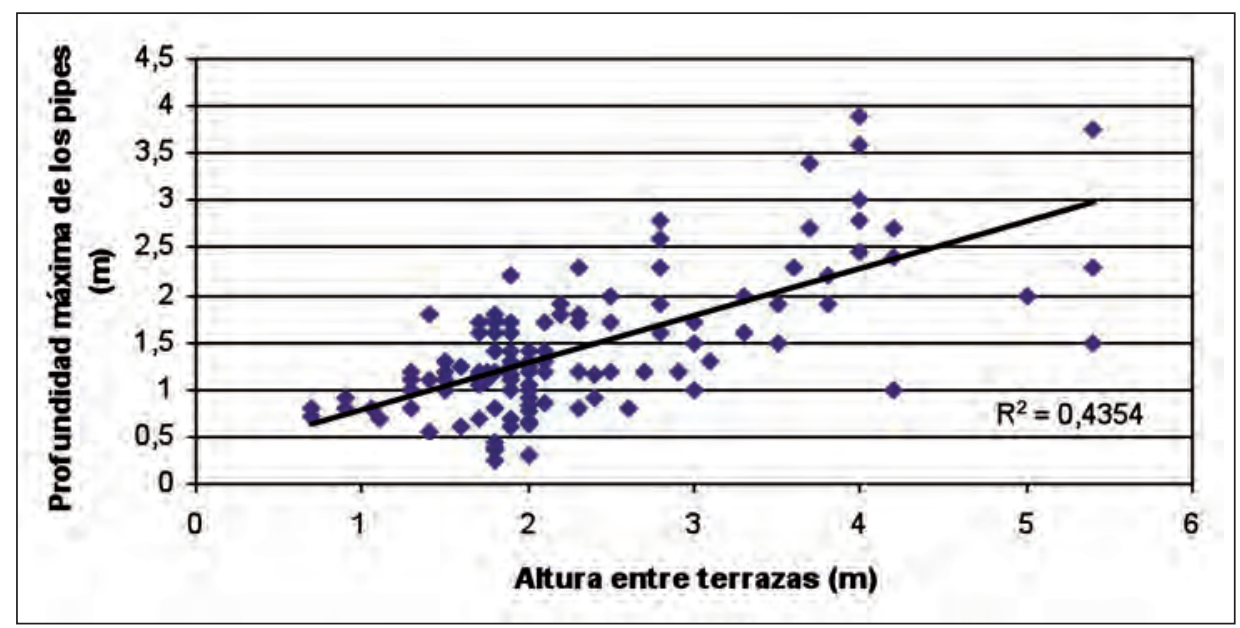

Figura 14. Relación entre altura de las terrazas y profundidad máxima de los pipes, con profundidad $=o>$ a la altura de la terraza.

\subsection{Relaciones entre variables}

Al relacionar los distintos parámetros analizados, tanto de las características topográficas de las parcelas, como de los pipes y los volúmenes de suelo perdido o tasas de erosión obtenidas, se muestran algunas correlaciones significativas.

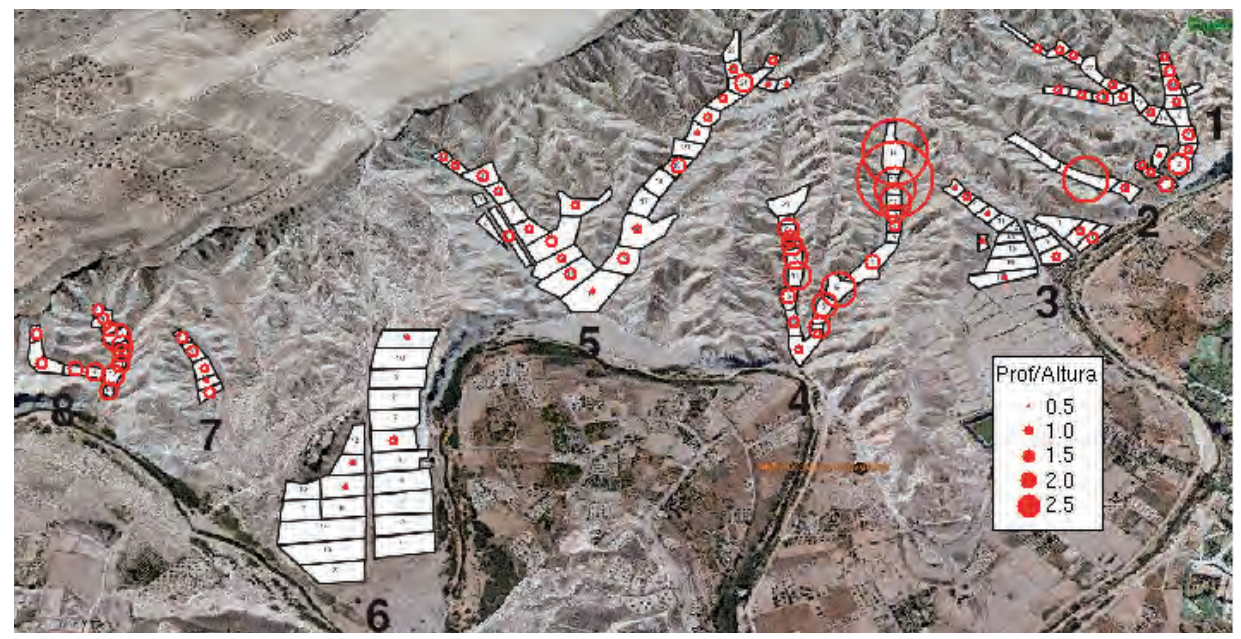

Figura 15. Profundidad máxima de los pipes/altura de la parcela. 


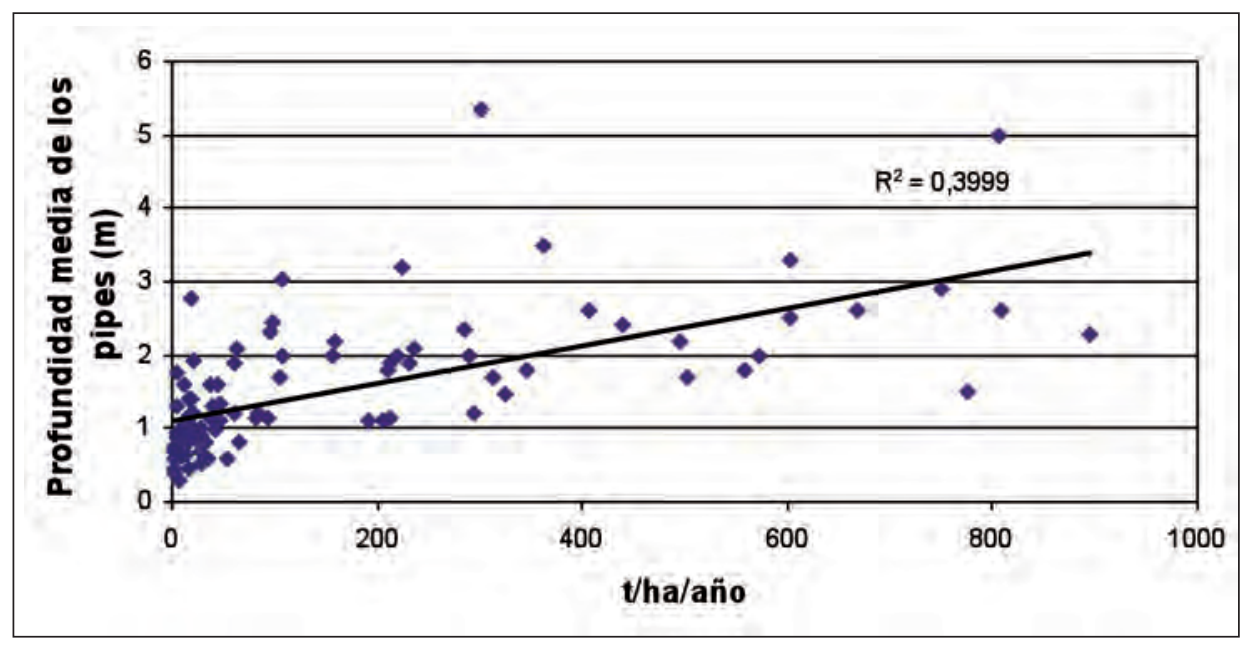

Figura 16. Relación entre profundidad media de los pipes y tasas de erosión.

Una correlación significativa, y que como es lógico resulta evidente, se muestra entre el porcentaje de superficie afectada por procesos de piping y el volumen de suelo perdido (Fig. 10), en donde a mayor superficie afectada por piping los volúmenes de suelo perdido son mayores.

Otras relaciones, aunque con menor coeficiente de correlación se hayan entre la superficie afectada por piping y las longitudes, profundidades y anchuras medias de los pipes, siendo la profundidad con la que mayor correlación se obtiene (Figs. 11, 12, 13)

Las relaciones entre superficie afectada por piping y diversos parámetros de las parcelas no muestran correlaciones altas, aunque se observa que la superficie afectada es mayor cuanto menor es la longitud y la anchura de la parcela. Es decir que cuanto menor es el tamaño de la parcela el grado de afección es mayor. Lo mismo ocurre al relacionar la superficie de la parcela con las pérdidas de suelo, a menor superficie las pérdidas de suelo se elevan.

Respecto a la relación entre altura de las terrazas y profundidad de los pipes, dependiendo del conjunto de datos que analicemos la correlación puede ser mejor o peor. La mejor correlación se haya si relacionamos la altura entre terrazas y las profundidades máximas o medias de aquellos pipes que tienen profundidades iguales o superiores a la altura de la terraza, cosa que ocurre cuando el nivel de base es el de dos terrazas consecutivas. En este caso cuanto mayor es la altura de la terraza mayor es la profundidad máxima o media de los pipes (Fig. 14) y, por tanto, el desarrollo de los procesos de piping es mayor. Es importante destacar como, en algunos casos, la nivelación de tierras y la construcción de terrazas, que suele ser una práctica habitual de conservación de suelos, se puede convertir en un gran 
problema, en el caso de que se den las circunstancias favorables para el desarrollo de procesos de piping (Faulkner, 2006; Romero Díaz et al., 2007).

A nivel espacial en la figura 15 se muestran las áreas en donde esta relación (altura de las terrazas/profundidad máxima de los pipes) es mejor, destacando la zona 4, 1 y 8.

Al relacionar la profundidad media de los pipes con las pérdidas de suelo o tasas de erosión también se encuentra una relación positiva, mostrando como las mayores pérdidas de suelo se producen en las áreas con pipes de profundidades medias más elevadas (Fig. 16).

\section{Conclusiones}

Como conclusión general se puede decir que, en el área estudiada, sí existe relación entre las características topográficas de las parcelas, los pipes y el desarrollo de procesos de piping y pérdidas de suelo. Se ha constatado como las áreas en las que se da un desarrollo mayor del piping y las mayores pérdidas de suelo se corresponden, en general, con las zonas de menor superficie, menor longitud y anchura de las parcelas. Así las zonas 7 y 8 , que son las que tienen la menor anchura media y máxima, son también unas de las zonas que registran las mayores pérdidas de suelo. Y las zonas en donde se alcanzan las mayores longitudes como son las zonas 5 y 6 se corresponden con aquellas zonas que menos pérdidas de suelo han registrado.

Los perfiles topográficos realizados de las distintas vaguadas aterrazadas muestran como parece existir una relación entre vaguadas cortas en longitud con importantes gradientes topográficos y desarrollo de piping.

El mayor número de pipes, no siempre se encuentra en las áreas que mayor afección superficial de piping poseen, debido al menor tamaño de los pipes o la escasa evolución de los mismos.

Las zonas con mayores pérdidas de suelo y tasas de erosión se corresponden con parcelas que poseen pipes de grandes dimensiones, tanto en longitud como en anchura y profundidad. La profundidad máxima que alcanzan algunos pipes es superior a la profundidad de la parcela en la que se encuentran, debido a que el pipe ha alcanzado el nivel de base de la parcela inferior o el nivel de base del río.

Las pérdidas de suelo estimadas en las diferentes zonas y que han sido evacuadas son muy elevadas, del orden de $3154 \mathrm{~m}^{3}$ o $4100 \mathrm{t}$. Destaca la zona 4 con unas pérdidas de suelo de $11750 \mathrm{~m}^{3}$ o 763,7 t y una tasa media de erosión de 413 o de 361 t/ha/año, según se considere un periodo de 30 o 40 años. Y a pesar de que los volúmenes de suelo perdido y las tasas de erosión alcanzan valores muy elevados, estos son inferiores a la realidad, al no haberse podido medir los conductos interiores. 
En la zona 4, la de mayor erosión, es en donde se ha medido el mayor desarrollo longitudinal total, medio y máximo de los pipes y las mayores anchuras medias y máximas.

Se ha hallado una correlación significativa y lógica entre el porcentaje de superficie afectada por procesos de piping y el volumen de suelo perdido, en donde a mayor superficie afectada por piping los volúmenes de suelo perdido son mayores. También existe relación entre la superficie afectada por piping y las longitudes, profundidades y anchuras medias de los pipes, siendo con la profundidad con la que mayor correlación se obtiene.

Las relaciones entre superficie afectada por piping y diversos parámetros de las parcelas no muestran correlaciones altas, aunque se observa que la superficie afectada es mayor cuanto menor es la longitud y la anchura de la parcela. Es decir, que cuanto menor es el tamaño de la parcela el grado de afección es mayor. Lo mismo ocurre al relacionar la superficie de la parcela con las perdidas de suelo, a menor superficie las pérdidas se elevan. Por otra parte, cuanto mayor es la altura de la terraza mayor es la profundidad máxima o media de los pipes y, por tanto, el desarrollo de los procesos de piping y las pérdidas de suelo son mayores.

Del estudio realizado se desprende la importancia de la conservación de los suelos y el no abandono de los campos agrícolas, pues el abandono se constata como un factor primordial de la aparición de piping.

Por otra parte, también los pipes, sobre estos tipos de suelos, se ven favorecidos por el aterrazamiento. En primer lugar, porque la escasa pendiente de las terrazas favorece la infiltración en lugar de la escorrentía superficial y, en segundo lugar, porque el gradiente de altura entre dos terrazas acelera la actividad mecánica del agua al crear un gradiente hidráulico.

Como recomendación general y a la vista de estos resultados, para nuevos acondicionamientos de tierras mediante aterrazado, sería conveniente realizar terrazas de pequeñas dimensiones y de pequeño desnivel entre ellas.

\section{Referencias bibliográficas}

Botscheck, J., Krause, S., Abel, T., SkowroneK, A., (2002). Piping and erodibility of loessic soils in Bergisches Land, Nordrhein-Westfalen. Journal of Plant Nutrition and Soil Science-Zeitschrift für Pflanzenernahrung und Bodenkunde 165: 241-246.

BRYAN, R., JONES, J. A. A., (1997). The significance of soil piping processes: inventory and prospect. Geomorphology, 20: 209-218.

FAULKNER, H., (2006). Piping hazard on collapside and dispersive soils in Europe. In Soil Erosion in Europe (Boardman, J., Poesen, J., Eds.). John Wiley \& Sons, pp. 537-562. 
FAulKner, H., SpIVEy, D., AleXANDER, R., (2000). The role of some site geochemical processes in the development and stabilisation of three badland sites in Almería, southern Spain. Geomorphology, 35: 87-99.

FAulKner H, AleXANDER, R., Wilson, B.R., (2003). Changes to the dispersive characteristics of soils along an evolutionary slope sequence in the Vera badlands, southeast Spain: implications for site stabilisation. Catena, 50: 243-254.

LÓPEZ-Bermúdez, F., RoMERO-DíAZ, A., (1989). Piping erosion and badland development in southeast Spain. Catena Supplement, 14: 59-73.

Naidu, R., Sumner, M.E., Rengasamy, P., (Eds.) (1995). Australian Sodic Soils: distribution, properties and management. CSIRO publication, $351 \mathrm{pp}$.

PARKER, C.G., (1964). Piping, a geomorphic agent in landform development of the drylands. In, Land Erosion, Precipitation, Hydrometry, Soil Moisture. Proceedings of the General Assembly of Berkeley, 19-31 August 1963. International Association of Scientific Hydrology, 65: 103-113.

Poesen J., Vandaele, K., Van Wesemael, B., (1996). Contribution of gully erosion to sediment production on cultivated lands and rangelands. In Erosion and Sediment Yield: Global and Regional Perspectives. Proceedings of the Exeter Symposium, July 1996. IAHS Publication No. 236: 251-256. IAHS, Wallingford.

Rengasamy, P., Greene, RSB., Ford, GW., Mehanni, A.H., (1984). Identification of dispersive behaviour and the management of red brown earths. Australian Journal of Soil Research 22: 413-431.

Romero Díaz, A., López Bermúdez, F. (1985)., Procesos de erosión en Cuencas Neógenas-Cuaternarias: La Cuenca de Mula. Guía de Itinerarios Geográficos de la Región de Murcia. Departamento de Geografía, Universidad de Murcia, pp. 83-97.

Romero Díaz, A., Marín SAnleandro, P., SÁnchez Soriano, A., BElmonte SERRATO, F., FAULKNER, H., (2007). The causes of piping in a set of abandoned agricultural terraces in southeast Spain. Catena 69: 282-293.

Romero Díaz, A., Plaza Martínez, J.F., Sánchez Soriano, A., Belmonte SerRATO, F., RuIZ SinOGA, J.D., (2009). Estimación de volúmenes de suelo perdido por procesos de erosión por piping. Sureste de España. En: Avances en estudios sobre Desertificación (Romero Díaz, A., Belmonte Serrato, F., Alonso Sarría, F., López Bermúdez, F., Eds.). EDITUM, Universidad de Murcia, pp. 403-407.

SÁnchez Soriano, A., Romero Díaz, A., Marín Sanleandro, P., (2003). Procesos de piping en campos de cultivo abandonados (Campos del Río, Murcia). En: Control de la erosión y degradación del suelo (Bienes, R., Marques, M.J., Eds.). IMIA, Madrid, pp. 625-629. 
Torri, D., Borselli, L., Calzolari, C., Yanez, MS., SAlvador SANChis, MP., (2002). Soil erosion, land use, soil qualities and soil functions: effect of erosion. En: Man and Soil at the Third Millennium, Vol. I (Rubio, JL., Morgan, RPC., Asins, S., Andreu, V., Eds). Editorial Geoforma, pp. 131-148, Logroño.

UChida, T., Kosugi, K., MizuYama, T., (2001). Effects of pipeflow on hydrological process and its relation to landslide: a review of pipeflow studies in forested headwater catchments. Hydrological Processes, 15: 2151-2174.

Verachtert, E., Van Den Eechhaut, M., Poesen, J., Deckers, J., (2010). Factors controlling the spatial distribution of soil piping erosion on loess-derived soils: A case study from central Belgium. Geomorphology, 118: 339-348.

WATTS, G., (1991). The relationship between soil piping and changing farming techniques on semiarid agricultural terraces. Proceeding of $20^{\text {th }}$ General Assembly of the International Union of Geodesy and geophysics at Viene. IAHS Publication 202: 81-89.

ZHU, T.X., (2003). Tunnel development over a 12 year period in a semi-arid catchment of the Loess Plateau, China. Earth Surface Processes and Landforms, 28: 507-525. 